\title{
A Feline Case of Isolated Fourth Ventricle with Syringomyelia Suspected to be Related with Feline Infectious Peritonitis
}

\author{
Masato KITAGAWA ${ }^{1)}$, Midori OKADA ${ }^{2)}$, Tsuneo $\mathrm{SATO}^{3)}$, Kiichi KANAYAMA ${ }^{4)}$ and Takeo SAKAI ${ }^{5)}$ \\ 1) Laboratory Comprehensive Veterinary Clinical Studies, Nihon University School of Veterinary Medicine, 1866 Kameino, Fujisawa, \\ Kanagawa 252-8510, 2) Pet Clinic ANIHOS, 1-14-11 Minamitokiwadai, Itabashiku, Tokyo 174-0072, \\ ${ }^{3)}$ Department of Veterinary Physiology, Nihon University School of Veterinary Medicine, 1866 Kameino, Fujisawa, Kanagawa 252-8510, \\ 4) Department of Veterinary Pathology, Nihon University School of Veterinary Medicine, 1866 Kameino, Fujisawa, Kanagawa 252-8510 and \\ ${ }^{5)}$ Department of Preventive Veterinary Medicine and Animal Health, Nihon University School of Veterinary Medicine, 1866 Kameino, \\ Fujisawa, Kanagawa 252-8510, Japan
}

(Received 11 May 2006/Accepted 14 March 2007)

ABSTRACT. A one-year-old female cat was unable to stand. Magnetic resonance imaging was performed, and an enlargement of the lateral, third, and fourth ventricles and syringomyelia were detected. The cat was diagnosed with an isolated fourth ventricle (IFV) with syringomyelia. The serum isoantibody test for the feline infectious peritonitis (FIP) virus was 1:3,200. After the cat died, a pathological examination revealed nonsuppurative encephalomyelitis. We suspected that the IFV, detected in the cat, was associated with FIP encephalomyelitis. To our knowledge, there has been no report on IFV in veterinary medicine.

KEY WORDS: feline, isolated fourth ventricle, syringomyelia.

J. Vet. Med. Sci. 69(7): 759-762, 2007

Isolated fourth ventricle (IFV) occurs when the fourth ventricle communicates with neither the third ventricle nor the basal cisterns due to blockage of the sylvian aqueduct and outlet of the fourth ventricle. In human medicine, IFV commonly occurs following shunting treatment for hydrocephalus $[6,17]$. In veterinary medicine, to our knowledge, there has been no report regarding IFV. Syringomyelia is a disease that causes cavities in the spinal cord and may be associated with neoplasm, anomaly, arachnitis, trauma, etc [1]. Moreover, it has been reported that in human medicine, syringomyelia is caused secondary to IFV [10]. Here, we report the case of a cat having IFV with syringomyelia suspected to be related with feline infectious peritonitis (FIV) encephalomyelitis.

A one-year-old female cat with a body weight of $2.64 \mathrm{~kg}$ was unable to stand following treatment for ataxia at a private hospital. The postural reaction test revealed absence in the left limb and the right pelvic limb and decrease in the right thoracic limb. The menace reaction had also decreased. Moreover, the cat was unwilling to stretch her neck. The cat was admitted to our hospital (Nihon University Animal Medical Center) for a detailed examination. She exhibited anorexia, loss of body weight, and tetraplegia. Neurological examination revealed that all the limbs showed a decreased response in the postural reaction test; however, no abnormalities were noted in the cranial nerve test. We suspected that the cat had a lesion in the cranial and/or cervical area and hence, we performed magnetic resonance imaging (MRI) (0.5 Tesla, FlexArt, Toshiba, Tokyo)

\footnotetext{
* Correspondence to: Kitagawa, M., Laboratory Comprehensive Veterinary Clinical Studies, Nihon University School of Veterinary Medicine, 1866 Kameino, Fujisawa, Kanagawa 252-8510, Japan.

e-mail:kitagawa@brs.nihon-u.ac.jp
}

and collected cerebrospinal fluid (CSF). The MRI revealed enlargement of the fourth ventricle and a mild enlargedment of the third and lateral ventricles (Fig. 1a, b, c). The images of inner wall of the fourth ventricle and the margin of the brain stem were enhanced by using a contrast agent (gadoteridol, $0.1 \mathrm{mmol} / \mathrm{kg}$ : ProHance ${ }^{\circledR}$, BRACCO International). Moreover, stenosis of the sylvian aqueduct, herniation of the cerebellum, and cervical syringomyelia were noted (Fig. 2a, b; arrow and arrowheads). The periphery of the ventricles and meninges showed hyperintensity on the fluid attenuated inversion recovery (FLARE) images (Fig. 3). CSF analysis revealed $423 \mathrm{mg} / \mathrm{d} l$ of protein, 280 cells $/ \mathrm{mm}^{3}$ (pleomonocytosis), and Pandy's test 4+. Serum isoantibody tests for the feline leukemia and feline immunodeficiency viruses were negative; however, that for the feline infectious peritonitis (FIP) virus was 1:3,200. The cat was hospitalized. Prednisolone (1 mg/kg, SC, q12h) and amoxicillin $(22 \mathrm{mg} / \mathrm{kg}$, $\mathrm{PO}, \mathrm{q} 12 \mathrm{~h}$ ) were administered to improve the appetite and increase the weight of the cat. However, the neurological signs did not improve. The cat exhibited cerebellar signs such as decerebellate rigidity (extension of the thoracic limbs and flexion of the pelvic limbs) and vertical nystagmus 20 days after the MRI. After the administration of mannitol $(0.5 \mathrm{~g} / \mathrm{kg}, \mathrm{IV}, \mathrm{q} 12 \mathrm{~h})$, furosemide $(0.7 \mathrm{mg} / \mathrm{kg}, \mathrm{IV}$, $\mathrm{q} 12 \mathrm{~h}$ ), and an isotonic solution, the cerebellum signs improved. However, since deterioration of the cerebellum signs and general condition including anorexia and anemia (PCV $42 \%$ to $23 \%$ ) persisted, syrinx-subarachnoid space shunting was performed 40 days after the MRI was performed. The cat died without recovery from general anesthesia.

Pathological examination revealed infiltration of lymphocytes and plasma cells and perivascular cuffing by monocytes in the spinal cord, cerebellum, medulla, and cerebrum. 

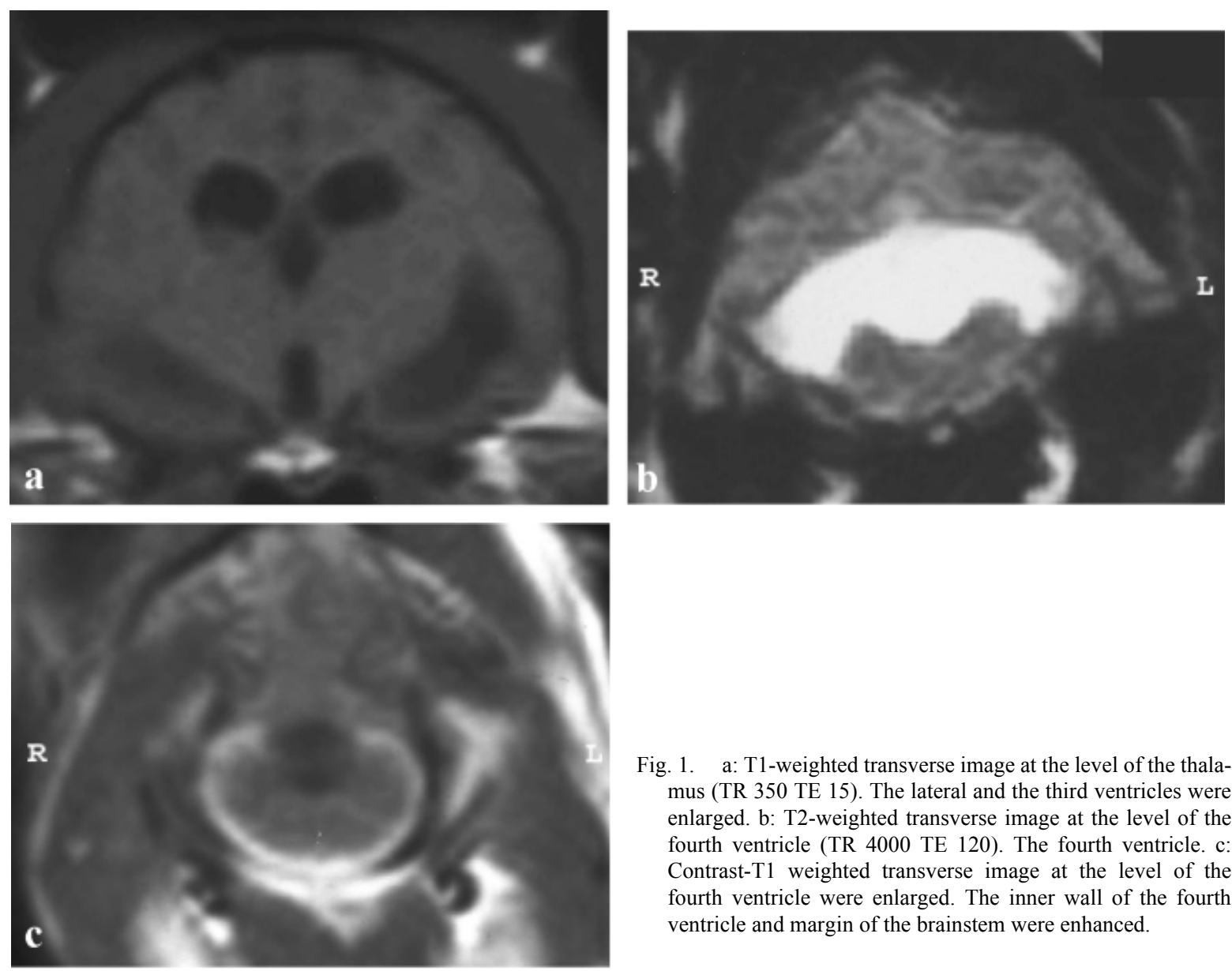

Fig. 1. a: T1-weighted transverse image at the level of the thalamus (TR 350 TE 15). The lateral and the third ventricles were enlarged. b: T2-weighted transverse image at the level of the fourth ventricle (TR 4000 TE 120). The fourth ventricle. c: Contrast-T1 weighted transverse image at the level of the fourth ventricle were enlarged. The inner wall of the fourth ventricle and margin of the brainstem were enhanced.

In the spinal cord, plasma cells also infiltrated the submeninges. In particular, the infiltration of lymphocytes and plasma cells was prominent in the fourth ventricle and ventral portions of the brainstem and aqueduct; mild infiltration was observed in the lateral periventricular region (Fig. 4a). Moreover, the aqueduct exhibited stenosis (Fig. 4b). However, most of the inner wall of the syrinx was destroyed by inflammation; some parts of it were lined by ependyma (Fig. 4c). The condition was diagnosed as nonsuppurative encephalomyelitis and meningitis.

It has been reported that the ventricles of a normal cat are too narrow to permit observation of the walls of the ventricles [9]. In the MRI findings of this cat, the ventricles were considered to be enlarged because the walls of all the ventricles were observed. However, the aqueduct was not observed, suggesting aqueduct stenosis or blockagede. Although enlargement of the fourth ventricle is observed when the outlet of the fourth ventricle is closed, the aqueduct is also simultaneously enlarged [18]. In this cat, it was suspected that enlargement of all the ventricles occurred due to blockage of flow in the aqueduct and the outlet of the fourth ventricle; this resulted in IFV.

The cat exhibited tetraparesis at the initial examination, following which vertical nystagmus and decerebellate rigid- ity became evident. It has been reported that decerebellate rigidity occurs due to injury of the rostral lobe of the cerebellum, and vertical nystagmus occurs due to injury of the cerebellum and/or the brain stem $[4,8]$. In humans, it has been reported that IVF might cause various signs and symptoms including cerebellar ataxia, incoordination, diplopia, nystagmus, facial paralysis, dysarthria, dysphasia, pyramidal pathways disorder, disturbance of consciousness, vomiting, respiratory disorder, etc. In this case, tetraparesis and cerebellar signs were detected. Although cerebellitis was observed pathologically, the cerebellar signs improved following the administration of intracranial decompressive agents (mannitol and furosemide). Accordingly, it was suspected that these signs were caused by pressure on the cerebellum due to the enlargement of the fourth ventricle; the effect on the brain stem was mild because no cranial nervous signs were detected.

It has been reported that the signs of IFV occurs due to the increasing pressure in the fourth ventricle [6]. In humans, IFV is occasionally accompanied with upward tentorial herniation because almost all patients with IFV receive shunting treatment for hydrocephalus. Further, it has been reported that IVF may be associated with tonsillar herniation (foraminal herniation) $[6,12]$. It has also been reported 

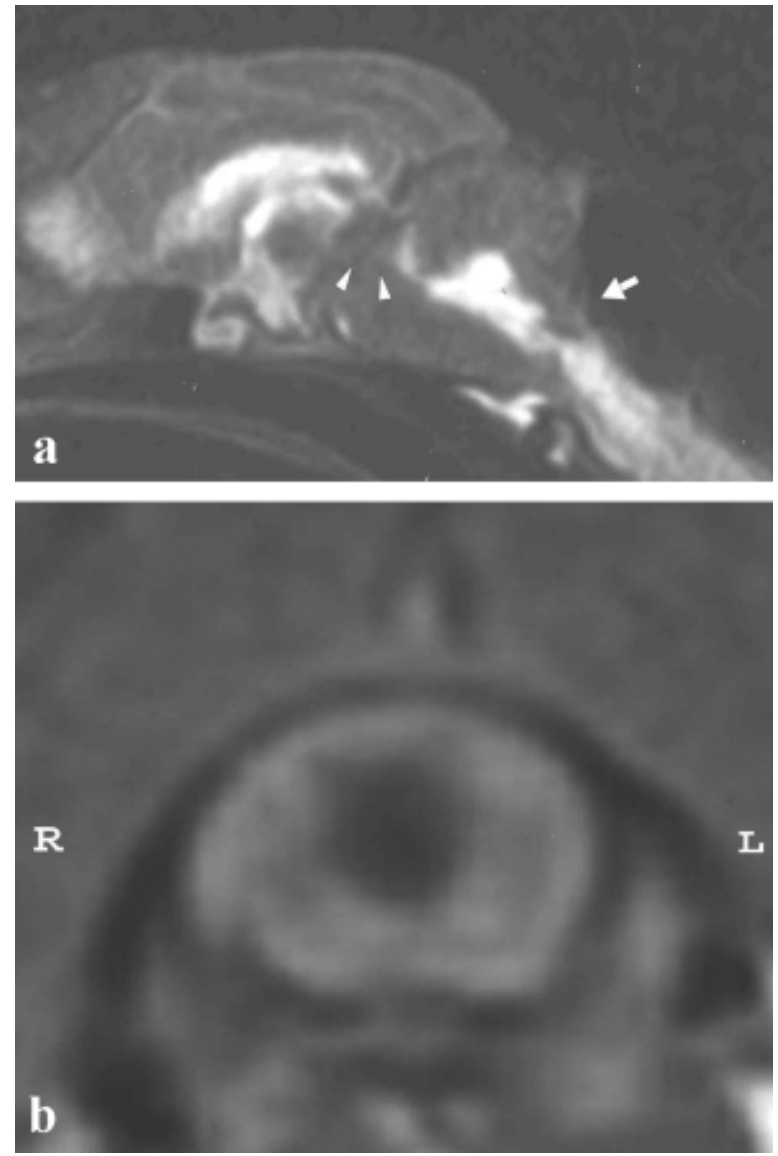

Fig. 2. a: T2-weighted sagittal image (TR 4000 TE 120). Occlusion of the aqueduct (arrow heads), enlargement of the fourth ventricle, and herniation of the cerebellum (arrow) are noted. Syringomyelia starting from the craniovertebral junction is observed. b: T1-weighted transverse image at the level of the neck (TR 350 TE 15). Syrinx is observed at the center of the spinal cord.

that although Chiari anomaly is a common cause of cerebellar herniation, space-occupying lesions in the posterior fossa may lead to tonsillar herniation [16]. It has also been reported that tonsillar herniation might be caused secondary to the increase in intracranial pressure [1]. In this case, it was suspected that tonsillar herniation was caused by the increasing pressure in the supratentorial and posterior fossae due to enlargement of the lateral and fourth ventricles.

In this case, medication caused a transient improvement in the general condition and neurological signs, but these signs subsequently deteriorated. Although the MRI and CSF examination were not repeated just before the operation, considering the preoperative clinical signs, we suspected that the hydrocephalus, meningoencephalitis, and meningitis deteriorated, and the cat died due to the effect of anesthesia or intense change in the intracranial pressure due to a shunt therapy.

There are few reports regarding feline syringomyelia $[2$, $3,11,12,18]$. Furthermore, these were cases of deforma-

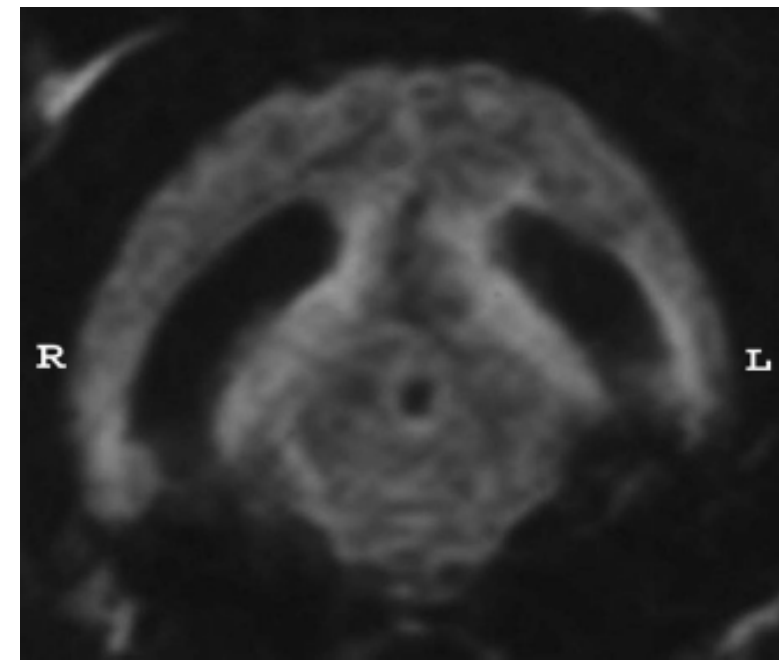

Fig. 3. FLARE transverse image at the level of the mid brain (TR 8000 TE 120). Hyperintense areas are noted in the periphery of the ventricles.

tion or hydrocephalus with blockage of the outlet of the fourth ventricle. It has been reported that syringomyelia following blockage of the outlet of the fourth ventricle was due to central canal enlargement (hydromyelia) that was caused by inflow from the ventricles $[13,18]$. Hence, we suspected that the syringomyerlia of this cat was due to a hydromyelia because hydrocephalus, enlargement of the fourth ventricle, and an ependymal lining the inner wall of the syrinx were detected.

It has been reported that the MRI findings of FIP encephalomyelitis are enlargement of the ventricles and an abnormal signal in the periphery of the ventricles [5]. Moreover, it has been reported that FIP is a common infectious disease causing feline hydrocephalus [7]. In this case, enlargement of the ventricles was detected and the margin of the brainstem and fourth ventricle were enhanced in the contrast-T1 weighted images. In CSF analysis of the cat with FIP, it has been reported that lymphocytic pleocytosis and protein levels exceeding $200 \mathrm{mg} / \mathrm{d} l$ may be observed (CSF protein does not usually exceed $100 \mathrm{mg} / \mathrm{d} l$ in infection diseases despite the occurrence of FIP) [14]. In this case, an increase in CSF protein level was detected. Pathological findings of FIP encephalomyelitis revealed ependymitis and epiventriculitis in the fourth ventricle, meningitis, choroiditis, and infiltration of lymphocytes and plasma cells [5]. Moreover, it has been reported that the degree of meningeal infilteration increased towards the ventral and caudal regions of the brain and was most severe at the base of the cerebellum and the brain stem, including the medulla oblongata [5]. In this case, there were lesions in the fourth ventricle, brainstem, and cerebellum, that were enhanced in the contrast T1 weighted images. The pathological examination revealed inflammation of these lesions. The ventral portion of the brainstem in particular was prominent. These findings resembled those of a previous report [5]. Based on the MRI 

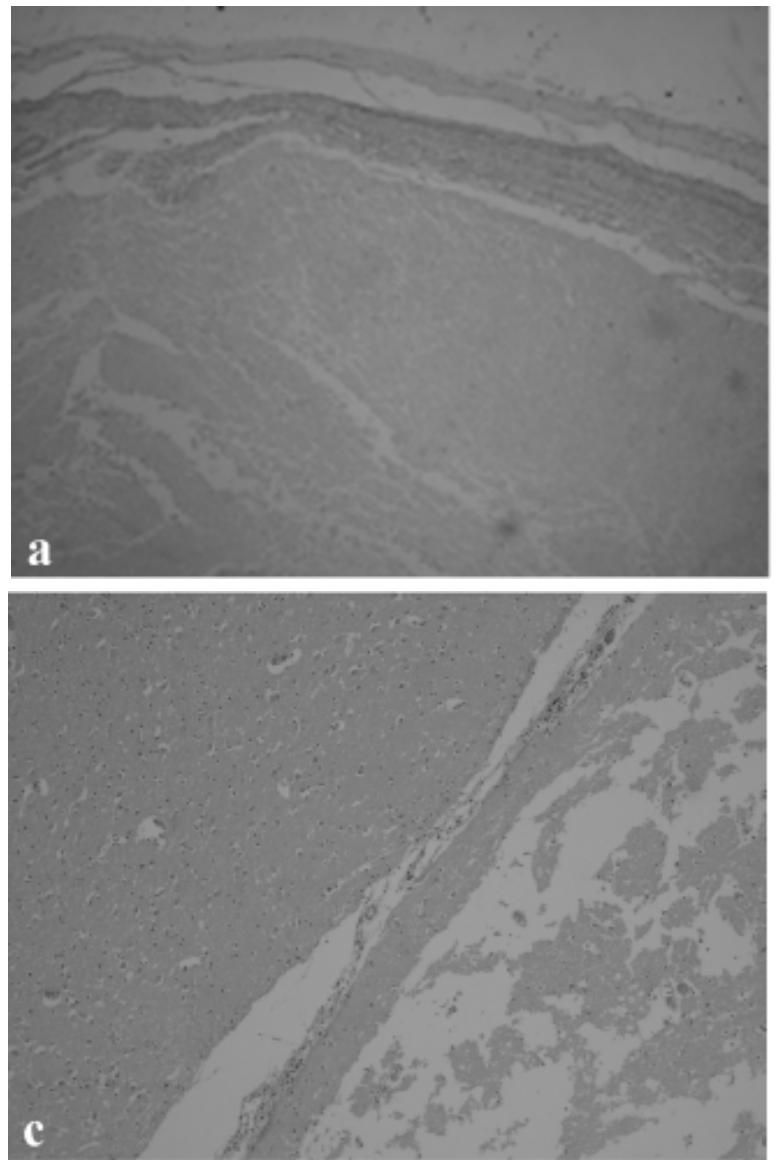

findings, pathological examination, and CSF analysis, it was suspected that in this cat, FIP encephalomyelitis caused stenosis of the outlet of the fourth ventricle and aqueduct and that the cat had IFV and syringomyelia. In human, postshunt isolated compartments were classified as isolated unilateral hydrocephaluse, isolated fourth ventricle, isolated rhombencephalic ventricle, and isolated central canal dilation [15]. Isolated rhombencephalic ventricle, characteristic of enlargement of the fourth ventricle and central canal, causes resultant from progressive of isolated fourth ventricle. In this case, it was considered that the condition of the cat was identical to that of isolated rhombencephalic ventricle, although the cause of each differs.

Here, we report the case of a cat having IFV with syringomyelia suspected to be related with feline infectious peritonitis (FIV) encephalomyelitis. This is the first such report, to the best of my knowledge.

\section{REFERENCES}

1. Bagley, R. S., Gavin, P. R., Silver, G. M., Moor, M. P., Kippenes, H. and Connors, R. 2000. Compend. Contin. Educ. Pract. Vet. 22: 471-479.

2. Bone, D. L. and Wilson, R. B. 1982. J. Am. Vet. Med. Assoc. 181: 928-929.

3. Clark, L. and Carlisle, C. H. 1975. Aust. Vet. J. 51: 392-394.

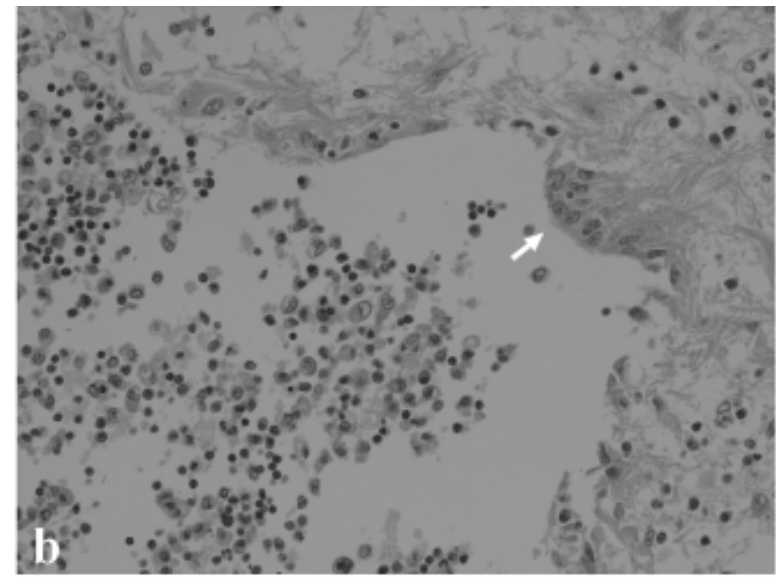

Fig. 4. Pathological findings of a: the brain stem $(\times 100)$, b: the aqueduct $(\times 400)$, and $c$ : syrinx of the spinal cord $(\times 100)$ (hematoxyline- eosin stain). Infiltration of lymphocytes and plasma cells are detected. The aqueduct exhibited stenosis. The ependyma is seen observed in the syrinx (arrow).

4. DeLahunta, A. 1983. Veterinary Neuroanatomy and Clinical Neurology, 2nd ed., Philadelphia, PA, Saunders.

5. Foley, J. E., Lapointe, J. M., Koblik, P., Poland, A. and Pedersen, N. C. 1998. J. Vet. Inter. Med. 12: 415-423.

6. Greenberg, M. S. 2001. Handbook of neurosurgery, 5th ed., Greenberg Graphics, Florida.

7. Harrington, M. L., Bagley, R. S. and Moore, M. P. 1996. Vet. Clin. North. Am. Small Anim. Pract. 26: 843-856.

8. Holliday, T. A. 1979/1980. Vet. Sci. Commun. 3: 259-278.

9. Hudson, L. C., Cauzinille, L., Kornegay, J. N. and Tompkins, M. B. 1995. Vet. Radiol. Ultrasound. 26: 267-275.

10. Kojima, N., Tamaki, N. and Matsumoto, S. 1988. No To Shinkei 40: 679-687.

11. Leipold, H. W., Huston, K., Blauch, B. and Guffy, M. M. 1974. J. Am. Vet. Med. Assoc. 164: 520-523.

12. Martin, A. H. 1971. Vet. Pathol. 8: 232-238.

13. Milhorat, T. H., Miller, J. I., Johnson, W. D., Adler, D. E. and Heger, I. M. 1993. Neurosurgery 32: 748-754.

14. Munana, K. R. 1996. Vet. Clin. North. Am. Small Anim. Pract. 26: $857-874$.

15. Oi, S., Kudo, H., Yamada, H., Kim, S., Hamano, S., Urui, S. and Matsumoto, S. 1991. J. Neurosurg. 74: 371-379.

16. Osborn, A. G. 1994. Diagnostic neuroradiology., Mosby, St. Louis.

17. Scotti, G., Musgrave, M. A., Fitz, C. R. and Harwood-Nash, D. C. 1980. Am. J. Roentgenol. 135: 1233-1238.

18. Tani, K., Taga, A., Itamoto, K., Iwanaga, T., Une, S., Nakaichi, M. and Taura, Y. 2001. J. Vet. Med. Sci. 63: 1331-1334. 